\title{
In Situ Atomic-Scale Observation of Dislocation Climb and Grain Boundary Transformation in Nanostructured Metal
}

\section{Shufen Chu}

Shanghai Jiao Tong University

\section{Pan Liu}

State Key Laboratory of Metal Matrix Composites, School of Materials Science and Engineering, Shanghai Jiao Tong University

\section{Yin Zhang}

Georgia Institute of Technology

\section{Xiaodong Wang}

Shanghai Jiao Tong University

\section{Shuangxi Song}

Shanghai Jiao Tong University https://orcid.org/0000-0003-2425-8084

\section{Ting Zhu}

Georgia Institute of Technology https://orcid.org/0000-0001-8612-9689

\section{Ze Zhang}

Zhejiang University

\section{Xiaodong Han}

Beijing Key Laboratory of Microstructure and Property of Advanced Materials, Beijing University of Technology, 100124 Beijing, China. https://orcid.org/0000-0002-3353-3802

\section{Baode Sun}

Shanghai Key Lab of Advanced High-temperature Materials and Precision Forming, School of Materials Science and Engineering, Shanghai Jiao Tong University, Shanghai 200240, China

\section{Mingwei Chen ( $\nabla$ mwchen@jhu.edu )}

Tohoku University https://orcid.org/0000-0002-8274-3099

\section{Article}

Keywords: atomic-scale observations, grain boundary transformation, dislocation climb, nanostructured metal

Posted Date: August 11th, 2021

DOl: https://doi.org/10.21203/rs.3.rs-753595/v1 
License: (c) (i) This work is licensed under a Creative Commons Attribution 4.0 International License. Read Full License

Version of Record: A version of this preprint was published at Nature Communications on July 18th, 2022. See the published version at https://doi.org/10.1038/s41467-022-31800-8. 


\section{In Situ Atomic-Scale Observation of Dislocation Climb and Grain Boundary Transformation in Nanostructured Metal}

Shufen $\mathrm{Chu}^{1}$, Pan Liu ${ }^{1,2 *}$, Yin Zhang ${ }^{3}$, Xiaodong Wang ${ }^{1}$, Shuangxi Song ${ }^{1}$, Ting Zhu $^{3 *}$, Ze Zhang ${ }^{4}$, Xiaodong Han ${ }^{5}$, Baode Sun ${ }^{1}$, Mingwei Chen ${ }^{2,6 *}$

${ }^{1}$ Shanghai Key Laboratory of Advanced High-temperature Materials and Precision Forming, State Key Laboratory of Metal Matrix Composites, School of Materials Science and Engineering, Shanghai Jiao Tong University, Shanghai, 200240, China ${ }^{2}$ WPI Advanced Institute for Materials Research, Tohoku University, Sendai 980-

$$
\text { 8577, Japan }
$$

${ }^{3}$ Woodruff School of Mechanical Engineering, Georgia Institute of Technology,

$$
\text { Atlanta, GA, 30332, USA }
$$

${ }^{4}$ Center of Electron Microscopy and State Key Laboratory of Silicon Materials, School of Materials Science and Engineering, Zhejiang University, Hangzhou

$$
\text { 310027, China }
$$

${ }^{5}$ Institute of Microstructure and Properties of Advanced Materials, Beijing University of Technology, Beijing, 100124, China

${ }^{6}$ Department of Materials Science and Engineering, Johns Hopkins University, Baltimore, MD, 21218, USA

*Corresponding authors: panliu@sjtu.edu.cn (PL); ting.zhu@me.gatech.edu (TZ); mwchen@jhu.edu (MC) 


\begin{abstract}
We report atomic-scale observations of grain boundary (GB) dislocation climb in nanostructured Au during in situ straining at room temperature. Climb of a dislocation occurs by stress-induced reconstruction of two atomic columns at the edge of an extra half atomic plane in the dislocation core. Different from the conventional belief of dislocation climb by destruction or construction of a single atomic column at the dislocation core, the new atomic route is demonstrated to be energetically favorable by Monte Carlo simulations. Our in situ observations also reveal GB transformation through dislocation climb, which suggests a means of controlling microstructures and properties of nanostructured metals.
\end{abstract}




\section{Introduction}

Nanostructured metals often exhibit superior mechanical properties due to strong size effects at the nanoscale ${ }^{1,2}$. They have been used as important components for modern nanotechnology and electronics ${ }^{3}$. In general, the nanoscale size of crystals affects the generation, motion, reaction and annihilation of dislocations ${ }^{4-10}$. However, the atomic-scale mechanisms of dislocation movement in nanoscale structures remains elusive, particularly for climb of dislocations that involves their non-conservative motion out of original slip planes. Theoretical calculations and atomistic simulations have been performed to investigate dislocation climb over relevant time and temperature scales ${ }^{11-15}$. Mesoscale simulations based on discrete dislocation dynamics $^{16-20}$ and phase field ${ }^{21-24}$ methods have been also used to investigate dislocation climb. However, in situ atomic-scale experimental observations of dislocation climb have not been realized to our knowledge. Hence, the dynamic evolution of dislocation cores during their climbing is not clearly understood, and the nanoscale size effects on dislocation climb remain little known.

Here, we report in situ atomic-scale observations of dislocation climb at a tilt grain boundary $(\mathrm{GB})$ in a $\mathrm{Au}$ ligament within de-alloyed nanoporous $\mathrm{Au}$ with the facecentered cubic structure ${ }^{25}$. Climb of GB dislocations is driven by applied bending load at room temperature. As a result, most of climbing GB dislocations escape from the free surface of the ligament, leading to the transformation of a high-angle GB (HAGB) to a $\Sigma 3$ twin boundary (TB). The atomic-scale climbing processes of dislocation cores demonstrate a new route of non-conservative dislocation motion, which is different 
from conventional understanding.

\section{Results}

Dislocation climb and GB transformation. In situ straining experiment was conducted using a double-tilt actuator inside a transmission electron microscope $(\text { TEM })^{26}$. High resolution TEM (HRTEM) images were recorded at a high rate of 30 frames per second, which greatly facilitates the tracking of step-by-step movements of individual GB dislocations. Figure. 1a shows the atomic structure of an Au ligament of diameter $\sim 7 \mathrm{~nm}$ before loading (time $t=0 \mathrm{~s}$ ). The reconstructed atomic model of the ligament is shown in Fig. 1a'. The ligament consists of three nano-sized grains, with a $\Sigma 3 \mathrm{~TB}$ between the left and middle grains and a HAGB between the middle and right grains. All three grains are aligned along the $[\overline{1} 10]$ zone axis. Simulated atomic images indicate that dark spots in HRTEM images correspond to $\mathrm{Au}$ atomic columns (Supplementary Fig. 1). The HAGB consists of an array of edge dislocations aligned vertically above each other. These GB dislocations have the identical Burgers vector of $1 / 2<011>\{111\}$, as determined from the Burgers circuit analysis in the inset of Fig. 1a. The core of each dislocation is marked by a white $\perp$ symbol placed right below the extra half-plane in Fig. 1a and by red atomic columns in Fig. 1a'. The GB dislocations exhibit negligible core dissociation which may arise from the geometrical constraints from small dislocation spacings, grain sizes and large applied stresses, despite a low stacking fault energy of $\mathrm{Au}\left(32-50 \mathrm{~mJ} / \mathrm{m}^{2} 27,28\right)$. Each GB dislocation in Fig. 1a is assigned with a number between " 1 " and "9" to facilitate the tracking of their 
movements. These dislocations distribute uniformly with an average spacing $d$ of 0.68 nm. The angle $\theta$ between the respective (111) plane in the middle and right grains is measured to track the change of misorientation angle between the two grains. Because $\theta$ is induced by the dislocation array, it obeys the Frank-Bilby equation ${ }^{29}$, $\theta=2 \arcsin \frac{b}{2 d^{d}}$, where $b$ is the Burgers vector length $(0.288 \mathrm{~nm}$ for Au). During in situ testing, the Au ligament was subjected to an effective bending moment, as indicated in Fig. 1a'. As a result, non-uniform normal stresses were exerted on the HAGB aligned approximately with the cross section of the ligament, and they will be shown later by strain mapping through the geometric phase analysis (GPA) ${ }^{30}$.

The HRTEM images in Fig. 1b-g reveal the dynamic rearrangement of dislocations "1" and "9" at the HAGB. Details of GB dislocation movements between these HRTEM images can be seen in Supplementary Fig. 2-4 and Movie 1. In Fig. 1b-g, the left TB is not included for clarity, as it remains nearly intact throughout in situ testing. The atomic configurations reconstructed from these HRTEM images are shown in Fig. 1h, where the $\theta$ value is given for each frame. During in situ testing, $\theta$ decreased gradually (Supplementary Fig. 5), giving a total rotation of $\sim 24^{\circ}$ between the adjoining grains across the HAGB. From $0 \mathrm{~s}$ (Fig. 1a) to $4.7 \mathrm{~s}$ (Fig. 1b), the upmost dislocation “9” moved up one atomic spacing out of its original $\{111\}$ slip plane. This nonconservative dislocation motion, called positive climb, resulted in removal of one atomic column at the dislocation core, causing a decrease of $\theta$ to $23.7^{\circ}$. From $4.7 \mathrm{~s}$ to 63.1 s, positive climb occurred for dislocations "7" to "9" (Fig. 1c and Supplementary Fig. 2). These climb activities resulted in further decrease of $\theta$ to $21.3^{\circ}$ and also led to 
annihilation of dislocation "9" from the top surface of the ligament. We usually observed the climb of a single dislocation at a time, while other neighboring dislocations remained stationary. From $63.1 \mathrm{~s}$ to $169.0 \mathrm{~s}$ (Fig. 1d), positive climb occurred for dislocations " 4 " to " 8 ", leading to annihilation of " 8 " from the top surface of the ligament. These GB dislocations also glided occasionally along the horizontal $\{111\}$ slip planes (Supplementary Fig. 2-3), so that the vertical alignment of GB dislocations in Fig. 1d changed compared with that in Fig. 1c. From $169.0 \mathrm{~s}$ to $177.4 \mathrm{~s}$ (Fig. 1e), dislocations "6" and "7" further climbed upward, leading to annihilation of dislocation "7" from the top surface of the ligament. As a result, the GB became close to a TB with a sharp interface in Fig. 1e. During this interval, dislocation "1" moved down one atomic spacing out of its original $\{111\}$ slip plane, called negative climb. From $177.4 \mathrm{~s}$ to $179.3 \mathrm{~s}$ (Fig. 1f), only dislocations "5" and "4" were left at the GB. The detailed annihilation process of dislocation " 6 " was not captured. This dislocation likely underwent positive climb and escaped the ligament from its top surface, similar to dislocations "7" to "9". Note that dislocations "1" to "3" glided along the horizontal $\{111\}$ planes in the middle grain, transmitted across the GB, and further glided into the interior of the right grain (Supplementary Fig. 2-4 and Supplementary Fig. 6). As a result, the local TB migrated to the right. At 180.7 s (Fig. 1g), dislocations "5" and "4" disappeared. Successive HRTEM images in Supplementary Fig. 7a-d indicate that dislocations "5" and "4" underwent positive climb and escaped the ligament from its top surface. After the entire array of 9 GB dislocations disappeared, the HAGB at $0 \mathrm{~s}$ became a coherent TB at $180.7 \mathrm{~s}$, with the concomitant formation of a thin twin lamella 
of four atomic layers thick between the newly formed TB and the preexisting TB (Fig. $1 \mathrm{~g}$ and Supplementary Fig. 4).

During the above in situ straining experiment, climb of GB dislocations occurred predominantly, resulting in plastic bending deformation of the ligament with a drastic decrease of grain misorientation by $\sim 24^{\circ}$. The non-conservative movements of GB dislocations were driven by the bending-induced normal stresses on the HAGB plane, being compressive in the upper part and tensile in the lower part of the ligament, as shown by the GPA maps in Supplementary Fig. 8. The positive climb of GB dislocations removed atomic columns at the dislocation cores so as to accommodate the compressive stresses on the HAGB plane, while the negative climb of GB dislocations introduced atomic columns to accommodate the tensile stresses. The local normal stress is estimated to be $\sim 3.2 \mathrm{GPa}$ based on the corresponding maximum lattice strain of $\sim 4 \%$ indicated by the strain profile in Supplementary Fig. $8 \mathrm{~b}$ and Young's modulus of $\sim 80$ GPa for Au. The large normal stress, together with the constrains from GBs, leads to the negligible dissociation of the $1 / 2<011>$ dislocations despite the low stacking fault energy of Au. The neutral plane with zero local strain is closer to the bottom surface of the ligament. Hence, a major portion of the HAGB (i.e., above the neutral plane) was subjected to compressive stresses, driving the positive climb of dislocations " 4 " to " 9 ". Dislocations "6" to "9" climbed before dislocations "4" and "5", because they were at larger distances from the neutral plane and thus under higher compressive stresses for driving their climb. In contrast, dislocations " 1 " to " 3 " were located below/close to the neutral plane and thus under relatively low tensile stresses. Hence, they exhibited the 
conservative motion of glide into the right grain, with occasional negative climb to accommodate local deformation incompatibility. During these processes, slip transmission of dislocations "1" to "3" across the GB led to local GB migration. Such dislocation glide activities indicate the buildup of large local shear stresses. Overall, the applied bending load resulted in the transformation of a HAGB to a TB during in situ straining primarily through dislocation climb and annihilation to the top surface above the neutral plane, in conjunction with dislocation glide and transmission across the GB below the neutral plane.

Climb velocities measurement. The high frame rate of HRTEM imaging enables us to measure the velocity of climb for dislocations " 1 " to "9". The maximum climb velocity of individual dislocations is given in Supplementary Table 1. As described earlier, the positive climb of dislocations " 6 " to "9" occurred before dislocations " 4 " and " 5 ". The maximum climb velocities of the former group are lower than the latter. Such different velocities can be rationalized using the aforementioned Frank-Bilby equation. Assuming an approximately constant rate of change of $\theta$ during bending, the climb velocity should increase with increasing spacing between GB dislocations. As dislocations " 6 " to " 9 " left the ligament, the dislocation spacings were increased, resulting in higher climb velocities of dislocations " 4 " and " 5 ". In contrast to the positive climb of dislocations "4" to " 9 ", dislocations " 2 " and " 3 " were close to the neutral plane and subjected to low tensile stresses. As a result, their driving forces of climb were low, giving near-zero climb velocities. Dislocation "1" was below but 
further away from the neutral plane, thus exhibiting occasional negative climb. To gain a quantitative sense of climb velocities, we note that the core of dislocation " 4 " moved up $\sim 0.80 \mathrm{~nm}$ within $\sim 0.5 \mathrm{~s}$ (Fig. 2 and Supplementary Movie 2). Figure. $2 \mathrm{f}$ shows the corresponding displacement and velocity of dislocation "4" against time measured from the HRTEM images in Fig. 2a-e. Despite a late start of climb, dislocation "4" exhibited a high maximum climb velocity of $17.2 \mathrm{~nm} / \mathrm{s}$ and vanished at the top surface of the ligament within $\sim 1 \mathrm{~s}$ (Supplementary Fig. 4c-e).

Reconstruction of dislocation core during positive climb process. The above results demonstrate the predominant dislocation climb in a nanostructured metal at room temperature that plays an important role in bending deformation and the stress-induced GB transformation. Although several models have been proposed on the atomistic mechanisms of dislocation climb $b^{14,15,31-35}$, the real atomic processes have not been observed through in situ experiment. Our in situ observations (Fig. 3-4 and Supplementary Movie 3-4) captured the step-by-step movements of dislocation climb and particularly the corresponding destruction or construction process of an atomic column at the dislocation core. As an example, Fig. 3a-f show in situ HRTEM images of the positive climb of dislocation " 7 " by moving up one atomic spacing out of its original $\{111\}$ slip plane. Figure. $3 \mathrm{~g}$ shows the corresponding line profiles of normalized intensity across the dislocation core, as extracted from the rectangle area enclosing a $\{111\}$ atomic layer in Fig. 3a-f. While the interpretation of HRTEM contrast and associated line profiles can be difficult, the small sample thickness and 
minimized spherical aberration allow a semi-quantitative analysis of the HRTEM images, which is supported by simulated images where the number of atoms in a column (i.e., sample thickness) has direct correlation with contrast intensity (Supplementary Fig. 1). Importantly, the changes of intensity at the atomic columns (i.e., valleys) in Fig. $3 \mathrm{~g}$ indicate that the climbing process of a GB dislocation involves the reconstruction of two adjacent atomic columns at the dislocation core, rather than a single atomic column at the end of the extra half-plane. When a climb event began, the intensities at the two valleys in the profile $b$, as marked by green arrows, increased significantly in comparison with the profile $a$. As this GB dislocation continued to climb, the two valleys in the profiles $a$ and $b$ gradually merged into one valley in the profiles $c$ to $f$, indicating the two atomic columns merge into one at the dislocation core. Note that the location of the intensity peak between the two valleys in the profiles $a$ and $b$ was occupied by the newly formed valley in the profile $f$, while its neighboring valleys also adjusted their positions to accommodate these changes. Hence, 5 atomic columns in the profile $a$ transformed into 4 columns in the profile $f$, as the dislocation core moved up one atomic layer out of its original $\{111\}$ plane. This transformation reflects a drastic local lattice contraction, as marked with two black dashed lines in Fig. 3g, and thus accommodates the local compressive stress arising from the applied bending load. Simultaneous climb of dislocation " 7 " and dislocation " 8 " caused a decrease of $\theta$ from $22.6^{\circ}$ to $21.3^{\circ}$, as seen from Fig. $3 \mathrm{a}$ and Fig. $3 \mathrm{f}$. The same atomic processes at the cores of positively-climbing dislocations were observed (Supplementary Fig. 9 and Movie 5). Based on in situ observations in Fig. 3, Supplementary Fig. 10 shows the reconstructed 
atomic configurations at the core of a climbing dislocation along with simulated HRTEM images which give the same profile evolution as Fig. 3g. In addition, the intensity profiles of atomic columns were analyzed by treating bright spots (i.e., peaks) in the HRTEM images as atomic columns, giving the equivalent result of reconstruction of the core of a climbing GB dislocation (Supplementary Fig. 11).

To understand the atomic processes at the core of climbing GB dislocations, we performed Grand Canonical Monte Carlo (GCMC) simulations using an atomic model of Au ligament reconstructed from the HRTEM image. As shown in Supplementary Fig. 12, the left end of the reconstructed ligament was fixed and a bending load was applied to the right end. Figure. 3h-1 display the top views (in the first row of images) and side views (second row) of GCMC results in a GB region containing two neighboring dislocation cores (black boxed region in Supplementary Fig. 12), which experienced compressive stresses from the bending load. In the top views, two atomic columns at the core of each GB dislocation were directly involved in the simulated climbing process and thus colored in red; two neighboring atomic columns were colored in blue to serve as a reference. Since GCMC simulations allowed the removal and insertion of atoms to lower the system energy, local chemomechanical equilibrium was maintained the core of the climbing dislocation. At the beginning of dislocation climb, the compressive stress led to local lattice distortion with decreased distance between the two atomic columns in red (Fig. 3i), driving atoms in these two columns to move toward each other (Fig. 3j). Eventually, the two atomic columns merged into one column (Fig. 3k-1), resulting in the positive dislocation climb by one atomic layer. 
Meanwhile, the $\theta$ value decreased from $22.3^{\circ}$ (Fig. 3h) to $20.8^{\circ}$ (Fig. 31), which is comparable with our experimental results (Fig. 3a-f). As seen from the corresponding side views, merging of the two atomic columns at each dislocation core involved a series of unit processes of removing one atom from a pair of atoms along the direction of sample thickness, while the two adjacent atomic columns in blue were not involved. These GCMC results directly support our in situ HRTEM observations of positive climb of each dislocation core through merging of two atomic columns as an energetically favorable process. Moreover, the GCMC also reveals the highly localized processes of merging of atom pairs along the direction of sample thickness, which cannot be observed from the HRTEM images that only show the projection of all atomic layers along the $[\overline{1} 10]$ viewing direction. Apparently, to experimentally resolve the individual atom motion in climbing dislocation cores, a new TEM technique, such as such as atomic resolution electron tomography ${ }^{36}$, is required in future study.

\section{Reconstruction of dislocation core during negative climb process. Our HRTEM}

observations also capture the atomic process of negative climb of GB dislocations, which involves the insertion of an atomic column at the dislocation core. Figure. 4a-d show in situ HRTEM images of the negative climb of dislocation "1". Figure. 4e shows the corresponding line profiles of normalized intensity across the dislocation core, as extracted from the respective rectangle enclosing a $\{111\}$ atomic layer in Fig. 4a-d. When negative climb began, the intensity valley indicated by the right arrow in the profile $a$ increased dramatically in the profile $b$. Meanwhile, the intensity peak indicated 
by the left arrow in the profile $a$ gradually became a valley in the line profile $d$, corresponding to the formation of a new atomic column. This indicates the splitting of a single atomic column into two columns by atom diffusion to the edge of the extra half-plane. During this process, other neighboring valleys were shift to accommodate the newly inserted valley, i.e., atomic column. Hence, 4 atomic columns (valleys) in the profile $a$ transformed into 5 columns (valleys) in the profile $e$, as the dislocation core moved down one atomic layer from its original $\{111\}$ slip plane. This transformation reflected a drastic local lattice expansion, as marked with two dashed lines in Fig. 4e, and thus accommodated the local tensile stress arising from the bending load. Negative climb of GB dislocations was also observed in our GCMC simulations, as shown in Supplementary Fig. 13. The combined in situ HRTEM and GCMC results provide a solid basis for future studies of kinetic pathways of atom diffusion at the core of climbing dislocations. It should be noted that the core of GB dislocations in our work exhibits negligible dissociation. Such compact core structures facilitate the observed climbing processes. The climb of GB dislocations can become more complicated when they dissociate into partial dislocations and thus warrants further in situ study in the future.

\section{Discussion}

By tracking the intensity profile at a dislocation core during its climbing process, together with GCMC simulations, our results reveal the atomic processes of stressdriven climb of GB dislocations at room temperature. In contrast to the conventional 
model of dislocation climb by removal or insertion of a single atomic column at the edge of the extra half atomic plane, we find the merging of two atomic columns into one atomic column for positive climb and the splitting of one atomic column into two atomic columns for negative climb. This route could be more energetically favorable compared to diffusion along one atomic column, as supported by our GCMC simulations. These volume non-conservative processes are driven by the normal stress acting on the local GB plane. In practice, these processes would involve the diffusion of vacancies/atoms to/away from the jog pairs at the core of a dislocation line, and warrant further study in the future. In our work, dislocation climb at room temperature is mainly driven by applied high stresses enabled by nano-sized structures. The electron beam effect on the sample temperate increase was estimated to be less than $5 \mathrm{~K}$ (see Supplementary Discussion). Such a small temperature increase is insufficient to drive dislocation climb. When the bending load is applied on the Au ligament, the sub-10 nm size is beneficial to dislocation climb at room temperature because the free surface nearby can act as a highly efficient vacancy source. Although the pre-existing GB may also facilitate the room-temperature dislocation climb by fast diffusion paths, it appears not to play an essential role in the dislocation climb since lattice dislocation climb was also observed (Supplementary Fig. 7e-h).

In summary, the in situ TEM observations experimentally reveal the atomic process of dislocation climb in a FCC metal. Climb of a dislocation occurs by stressinduced reconstruction of two atomic columns at the edge of an extra half atomic plane in the dislocation core, different from the conventional single atomic column models. 
The finding of the room temperature dislocation climb by high velocities may offer new insights into the unique time-dependent properties of nanocrystalline materials, such as room-temperature creep and low-temperature grain coarsening, and room temperature superplasticity ${ }^{1,37-40}$. In the current prevailing models, these low-temperature timedependent properties are usually attributed to the fast diffusions at pronounced GBs in nanostructured materials. The dislocation climb induced GB evolution and migration, observed in our study, provides a new mechanism on the fast GB deformation, evolution and migration. Our results also suggest a new route to develop strong and ductile nanostructured metals by controlling dislocation climb and thus GB stability through pinning dislocations by chemical doping or promoting climb by introducing vacancies to enhance their strength and deformability at room temperature.

\section{Methods}

Preparation of nanoporous Au (NPG) film. A NPG film of $100 \mathrm{~nm}$ thick was prepared by electrochemical dealloying of $\mathrm{Ag}$ from a $\mathrm{Ag}_{65} \mathrm{Au}_{35}$ (at.\%) white $\mathrm{Au}$ leaf in a 70 vol $\% \mathrm{HNO}_{3}$ solution, which was followed by carefully rinsed with distilled water to remove the residual nitric acid. After few times washing, the NPG film was cut into pieces by a knife blade. The actual size of NPG thin films ranges between $50 \mu \mathrm{m}$ to 1 $\mathrm{mm}$. Under an optical microscope, we utilized the tweezer to clamp the strain actuator and scooped out the NPG film from the deionized water to transfer it to the straining actuator. Before inserting into the TEM chamber, the holder with the loaded NPG film sample was processed by vacuum drying and plasma cleaner to remove possible organic 
contamination. The as-prepared NPG film has three-dimensional nanopore channels and $\mathrm{Au}$ ligaments. The average size of $\mathrm{Au}$ ligaments is about $20 \mathrm{~nm}$ with a wide distribution range from $\sim 5$ to $35 \mathrm{~nm}^{25}$. The Au ligaments have the face-centered cubic structure with an average chemical composition of $\mathrm{Au}_{97} \mathrm{Ag}_{3}$ (at.\%), measured by TEM equipped with an energy dispersive X-ray spectrometer.

In situ TEM straining experiment. In situ bending test was carried out using a doubletilt straining holder (Bestron Science and Technology Co., LTD, Beijing). A miniature device is fixed on this custom-designed TEM sample holder to apply force and displacement by a strain actuator. The holder enables controllable bending, tension, compression deformation of the sample and a range of $\pm 25^{\circ} X$-tilt and $\pm 25^{\circ} Y$-tilt ${ }^{26}$. The NPG sample was used as a model system to study the climb mechanism in FCC nanostructured metals, because it has abundant ligaments with a wide size distribution from 5 to $35 \mathrm{~nm}^{25}$. Many grain boundaries are available in the ligaments of as-prepared NPG films. Thus, it is not difficult to identify a HAGB for in situ straining experiment. The NPG sample was subjected to a strain rate of $\sim 10^{-3} \mathrm{~s}^{-1}$ by controlling the actuator; meanwhile, the NPG sample was readily tilted to a desired crystallographic zone axis for atomic-scale observation.

In situ Cs-corrected HRTEM characterization. We used a $200 \mathrm{kV}$ JEM ARM200F electron microscope (JEOL) equipped with a spherical aberration corrector (CEOS $\mathrm{GmbH}$ ) for the objective lens system. With the optimized Cs correction value, the point- 
to-point resolution of the TEM images is better than 1.3 A. Before the atomic resolution images are recorded, the objective lens aberrations were measured by evaluating the Zemlin tableau of an amorphous area. The residual lens aberrations are as follows: spherical aberration $\mathrm{Cs}=-1.15 \mu \mathrm{m}(95 \%$ certificate). Atomic-scale deformation processes are recorded by a Gatan charge-coupled device (CCD) camera at a rate of 30 frames per second.

Grand Canonical Monte Carlo simulations. We performed atomistic Monte Carlo simulations to investigate the mechanisms of dislocation climb and grain boundary transformation. The simulations were performed using LAMMPS ${ }^{41}$ with an embeddedatom method potential of $\mathrm{Au}^{42}$. Based on the HRTEM image of the Au ligament in Fig. 1a, we set up the initial structure containing a $\sum 3$ TB on the left and a HAGB on the right, as shown in Supplementary Fig. 12. Periodic boundary condition was applied in the out-of-plane direction of $\langle 110>$. The initial structure was relaxed by the conjugate gradient method to reach a stress-free state. We used the Grand Canonical Monte Carlo $(\mathrm{GCMC})^{43}$ method to mimic the atomic diffusion processes at GB dislocations. This method allows us to perform conventional Monte Carlo moves and exchange GB atoms with an imaginary reservoir of atoms. To impose the bending load, we applied an incremental rotation to the right grain by 0.1 degree counterclockwise and then performed $10000 \mathrm{GCMC}$ steps at $300 \mathrm{~K}$. The atoms in the green box were fixed to maintain the mechanical load. Due to the imposed rotation of the right grain, compressive and tensile stresses were generated on the top and bottom parts of the 
HAGB plane, respectively. In the GCMC simulation, atoms can be removed or inserted in the system based on the energy difference and the sampling temperature. We repeated the loading process by incremental rotation plus 10000 GCMC steps 50 times, in order to simulate the continuous increase of bending load in the experiment. When a whole atom column disappeared at the core of a GB dislocation, the positive/upward climb by one atomic layer was completed. Similarly, the negative/downward climb of a GB dislocation was simulated by the GCMC method, showing the insertion of an atomic column at the dislocation core. In the GCMC method, the chemical potential in the imaginary reservoir is $-3.7 \mathrm{eV} /$ atom, which is set to be $0.2 \mathrm{eV}$ higher than the bulk cohesive energy to accelerate the simulated diffusion process.

\section{References:}

1. Meyers, M. A., Mishra, A. \& Benson, D. J. Mechanical properties of nanocrystalline materials. Prog. Mater. Sci. 51, 427-556 (2006).

2. Zhu, T. \& Li, J. Ultra-strength materials. Prog. Mater. Sci. 55, 710-757 (2010).

3. Lu, W. \& Lieber, C. M. Nanoelectronics from the bottom up. Nat. Mater. 6, 841850 (2007).

4. Chen, M., et al. Deformation twinning in nanocrystalline aluminum. Science 300, $1275-1277$ (2003).

5. Greer, J. R. \& Nix, W. D. Nanoscale gold pillars strengthened through dislocation starvation. Phys. Rev. B 73, 245410 (2006).

6. Shan, Z. W., Mishra, R. K., Syed Asif, S. A., Warren, O. L. \& Minor, A. M. 
Mechanical annealing and source-limited deformation in submicrometre-diameter $\mathrm{Ni}$ crystals. Nat. Mater. 7, 115-119 (2008).

7. Lu, L., Chen, X., Huang, X. \& Lu, K. Revealing the Maximum Strength in Nanotwinned Copper. Science 323, 607-610 (2009).

8. Zheng, H., et al. Discrete plasticity in sub-10-nm-sized gold crystals. Nat. Commun. 1, 144 (2010).

9. Greer, J. R. \& De Hosson, J. T. M. Plasticity in small-sized metallic systems: Intrinsic versus extrinsic size effect. Prog. Mater. Sci. 56, 654-724 (2011).

10. Zhu, Q., et al. Metallic nanocrystals with low angle grain boundary for controllable plastic reversibility. Nat. Commun. 11, 3100 (2020).

11. Weertman, J. Theory of steady-state creep based on dislocation climb. J. Appl. Phys. 26, 1213-1217 (1955).

12. Roy, S. \& Mordehai, D. Annihilation of edge dislocation loops via climb during nanoindentation. Acta Mater. 127, 351-358 (2017).

13. Fan, Y., Osetskiy, Y. N., Yip, S. \& Yildiz, B. Mapping strain rate dependence of dislocation-defect interactions by atomistic simulations. Proc. Natl. Acad. Sci. U.S.A. 110, 17756-17761 (2013).

14. Kabir, M., Lau, T. T., Rodney, D., Yip, S. \& Van Vliet, K. J. Predicting Dislocation Climb and Creep from Explicit Atomistic Details. Phys. Rev. Lett. 105, 095501 (2010). 15. Abu-Odeh, A., Cottura, M. \& Asta, M. Insights into Dislocation Climb Efficiency in FCC Metals from Atomistic Simulations. Acta Mater. 193, 1-10 (2020).

16. Mordehai, D., Clouet, E., Fivel, M. \& Verdier, M. Introducing dislocation climb by 
bulk diffusion in discrete dislocation dynamics. Philos. Mag. 88, 899-925 (2008).

17. Gao, Y., et al. Investigations of pipe-diffusion-based dislocation climb by discrete dislocation dynamics. Int. J. Plast. 27, 1055-1071 (2011).

18. Niu, X. H., Luo, T., Lu, J. F. \& Xiang, Y. Dislocation climb models from atomistic scheme to dislocation dynamics. J. Mech. Phys. Solids 99, 242-258 (2017).

19. Ayas, C., van Dommelen, J. A. W. \& Deshpande, V. S. Climb-enabled discrete dislocation plasticity. J. Mech. Phys. Solids 62, 113-136 (2014).

20. Gu, Y., Xiang, Y., Quek, S. S. \& Srolovitz, D. J. Three-dimensional formulation of dislocation climb. J. Mech. Phys. Solids 83, 319-337 (2015).

21. Geslin, P.-A., Appolaire, B. \& Finel, A. Multiscale Theory of Dislocation Climb. Phys. Rev. Lett. 115, 265501 (2015).

22. Ke, J., Boyne, A., Wang, Y. \& Kao, C. Phase field microelasticity model of dislocation climb: Methodology and applications. Acta Mater. 79, 396-410 (2014).

23. Geslin, P.-A., Appolaire, B. \& Finel, A. A phase field model for dislocation climb. Appl. Phys. Lett. 104, 011903 (2014).

24. Liu, P., et al. Point defect sink strength of low-angle tilt grain boundaries: A phase field dislocation climb model. Int. J. Plast. 119, 188-199 (2019).

25. Liu, P., et al. Time-resolved atomic-scale observations of deformation and fracture of nanoporous gold under tension. Acta Mater. 165, 99-108 (2019).

26. Han, X., Liu, P., Zhang, Y., Yue, Y. \& Zhang, Z. Device and method for measuring electromechanical properties and microstructure of nano-materials under stress state. U.S. Patent Application US8069733 (2011). 
27. Gallagher, P. \& Llu, Y. The diversity of stacking fault energy determinations and its significance. Acta Metall. 17, 127-137 (1969).

28. Cockayne, D., Jenkins, M. \& Ray, I. The measurement of stacking-fault energies of pure face-centred cubic metals. Philos. Mag. 24, 1383-1392 (1971).

29. Hirth, J. \& Lothe, J. Theory of Dislocations. 2nd ed, (Wiley, New York, 1982).

30. Hÿtch, M., Snoeck, E. \& Kilaas, R. Quantitative measurement of displacement and strain fields from HREM micrographs. Ultramicroscopy 74, 131-146 (1998).

31. Balluffi, R. W. Mechanisms of dislocation climb. Phys. Status Solidi 31, 443-463 (1969).

32. Clouet, E. Predicting dislocation climb: Classical modeling versus atomistic simulations. Phys. Rev. B 84, 092106 (2011).

33. Lau, T. T., Lin, X., Yip, S. \& Van Vliet, K. J. Atomistic examination of the unit processes and vacancy-dislocation interaction in dislocation climb. Scripta Mater. 60, 399-402 (2009).

34. Dutta, A., Bhattacharya, M., Gayathri, N., Das, G. C. \& Barat, P. The mechanism of climb in dislocation-nanovoid interaction. Acta Mater. 60, 3789-3798 (2012).

35. Sarkar, S., et al. Finding activation pathway of coupled displacive-diffusional defect processes in atomistics: Dislocation climb in fcc copper. Phys. Rev. B 86, 014115 (2012).

36. Wang, C., et al. Three-Dimensional Atomic Structure of Grain Boundaries Resolved by Atomic-Resolution Electron Tomography. Matter 3, 1999-2011 (2020).

37. Ames, M., et al. Unraveling the nature of room temperature grain growth in 
nanocrystalline materials. Acta Mater. 56, 4255-4266 (2008).

38. Darling, K. A., et al. Extreme creep resistance in a microstructurally stable nanocrystalline alloy. Nature 537, 378-381 (2016).

39. Mohamed, F. A. \& Li, Y. Creep and superplasticity in nanocrystalline materials: current understanding and future prospects. Mater. Sci. Eng., A 298, 1-15 (2001).

40. Lu, L., Sui, M. L. \& Lu, K. Superplastic Extensibility of Nanocrystalline Copper at Room Temperature. Science 287, 1463 (2000).

41. Plimpton, S. Fast parallel algorithms for short-range molecular dynamics. $J$. Comput. Phys. 117, 1-19 (1995).

42. Ackland, G., Tichy, G., Vitek, V. \& Finnis, M. Simple N-body potentials for the noble metals and nickel. Philos. Mag. A 56, 735-756 (1987).

43. Frenkel, D. \& Smit, B. Understanding molecular simulation: from algorithms to applications. (Elsevier, 2001). 


\section{Acknowledgements}

P.L. is supported by Natural Science Foundation of China (51821001, 11704245), Natural Science Foundation of Shanghai (19ZR1475200, 21ZR1431200), and the Eastern Scholar Program at Shanghai Institutions of Higher Learning. M.C. is sponsored by National Science Foundation (NSF-DMR-1804320).

\section{Author contributions}

M.C. conceived and supervised the project. P.L. designed the experiments. S. C., and P. L. conducted the in situ Cs-corrected HRTEM experiments under the guidance of X. D. H and Z. Z. Y. Z. and T. Z. performed Monte Carlo simulations. S. C., P. L., T. Z., and M. W. C. analysed the data and wrote the manuscript. All authors contributed to the extensive discussions of the results.

\section{Competing interests}

The authors declare no competing interests. 

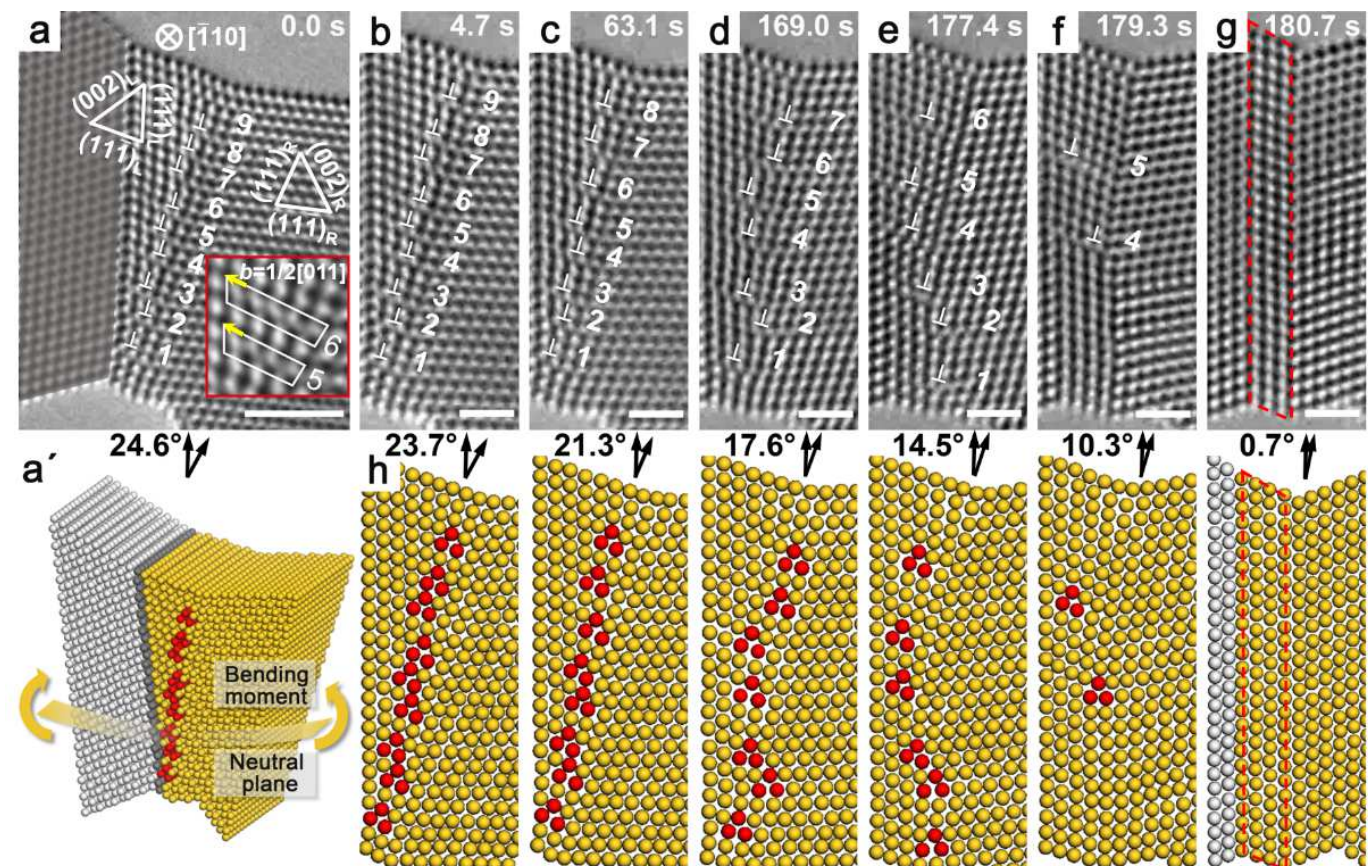

$0.7^{\circ}$

Figure 1. Representative in situ HRTEM images showing dislocation climb at a

HAGB and atomic models reconstructed from the HRTEM images. (a-g)

Dislocations are marked by both symbols $\perp$ and numbers. Burgers circuits in the inset of (a) indicate all the edge dislocations have an identical Burgers vector of 1/2[011] (11) $)$. The newly-formed twin lamella is indicated by red dashed lines in (g). Scale bar: (a) $2 \mathrm{~nm}$ and (b-g) $1 \mathrm{~nm}$. (a') Atomic model reconstructed from the HRTEM image in (a). The pre-existing $\Sigma 3 \mathrm{~TB}$ is marked by a dark grey plane and the dislocation cores are highlighted by red atomic columns. The yellow curved plane indicates the neutral plane of bending. (h) Reconstructed atomic configurations corresponding to HRTEM images in (b)-(g). 

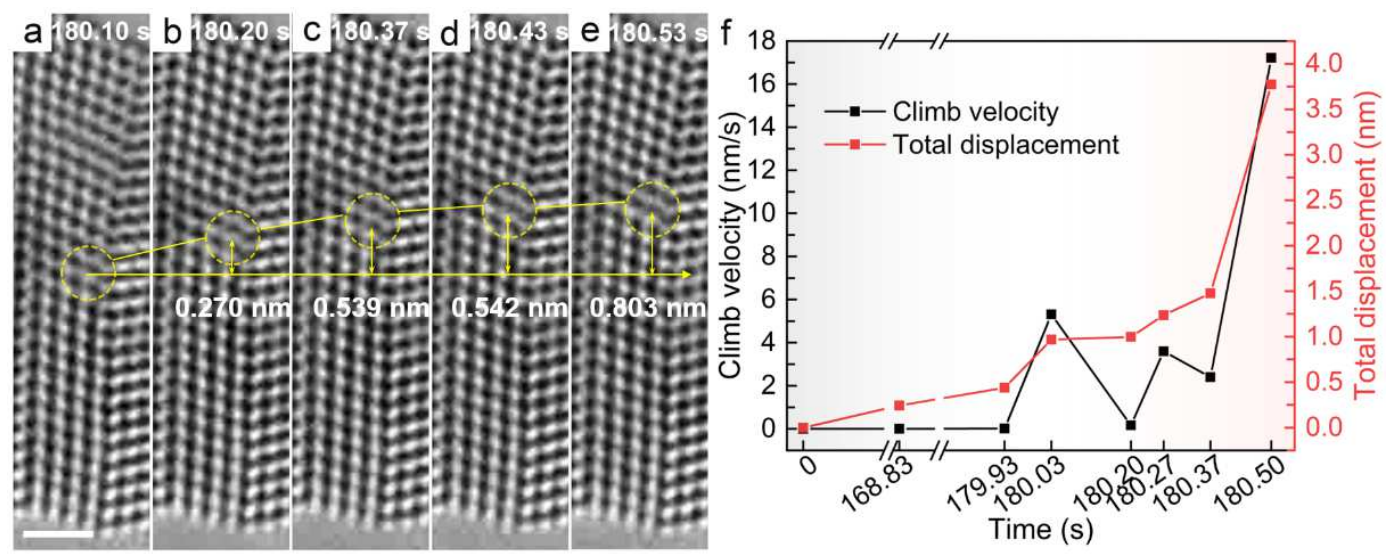

Figure 2. Climb velocity and displacement of dislocation "4" measured from the

HRTEM images. (a)-(e) HRTEM images showing fast movement of the core (circled by dashed lines). Scale bar: $1 \mathrm{~nm}$. (f) Climb velocity and displacement of dislocation "4" as a function of time during the whole in situ straining process. 


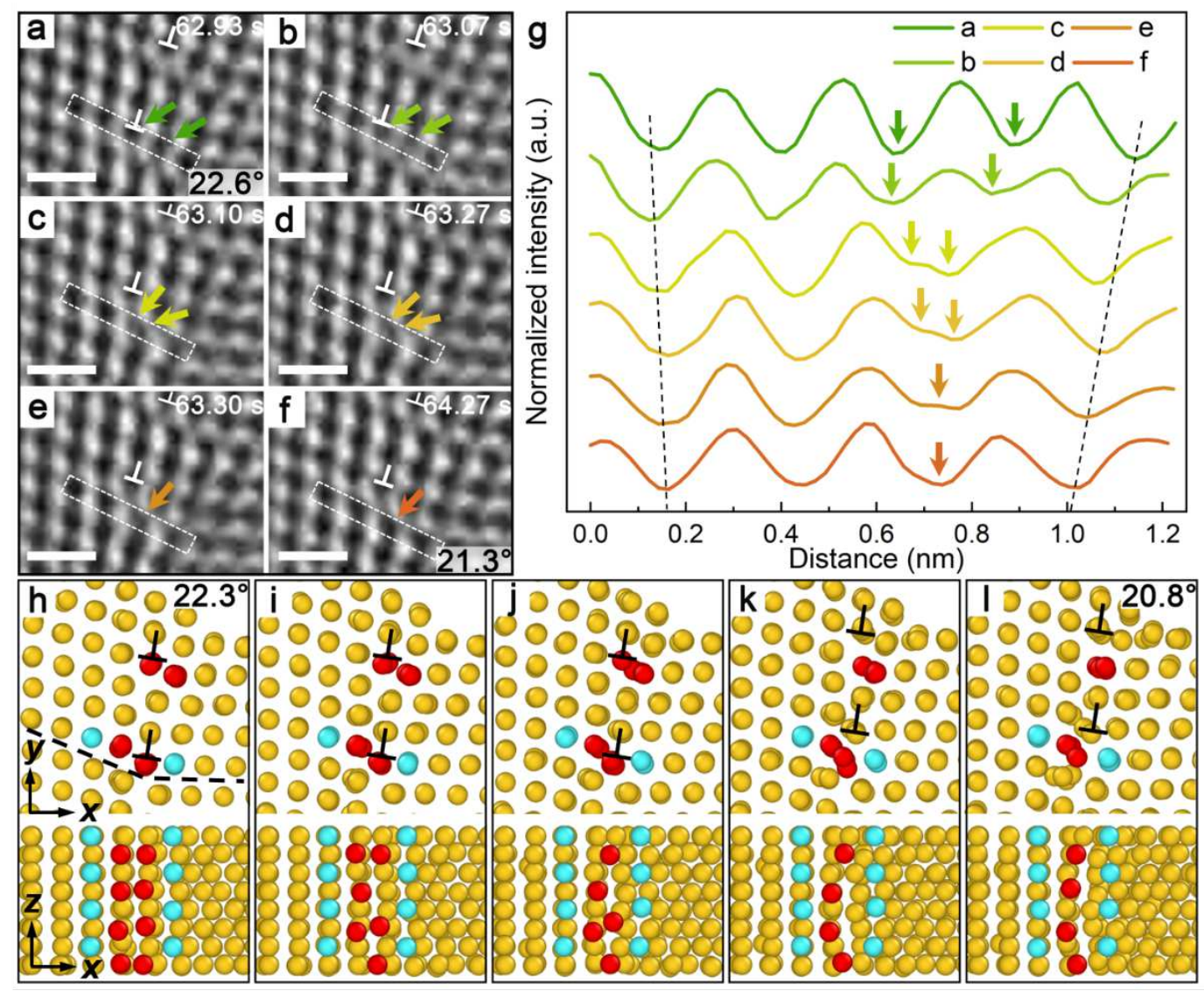

Figure 3. Reconstruction of dislocation cores during positive climb process. (a)-(f)

A series of HRTEM images showing the reconstruction of a dislocation core, with the corresponding contrast intensity profiles in $(\mathrm{g})$ extracted along the dotted rectangle areas in (a)-(f). Scale bar: $0.5 \mathrm{~nm}$. (h)-(l) GCMC results revealing the atomic structure evolution of dislocation cores during positive climb. The upper row of images shows the top view ( $x-y$ plane) of the merging process of two adjacent atomic columns (colored in red) at the core of each of two GB dislocations (marked by symbol $\perp$ ). The lower row shows the corresponding side view ( $x-z$ plane) generated by cutting the atomic structure along the black dotted line in (h). Another two neighboring atomic columns (colored in blue) serve as a reference and are not directly involved in the reconstruction process at the climbing dislocation core. 

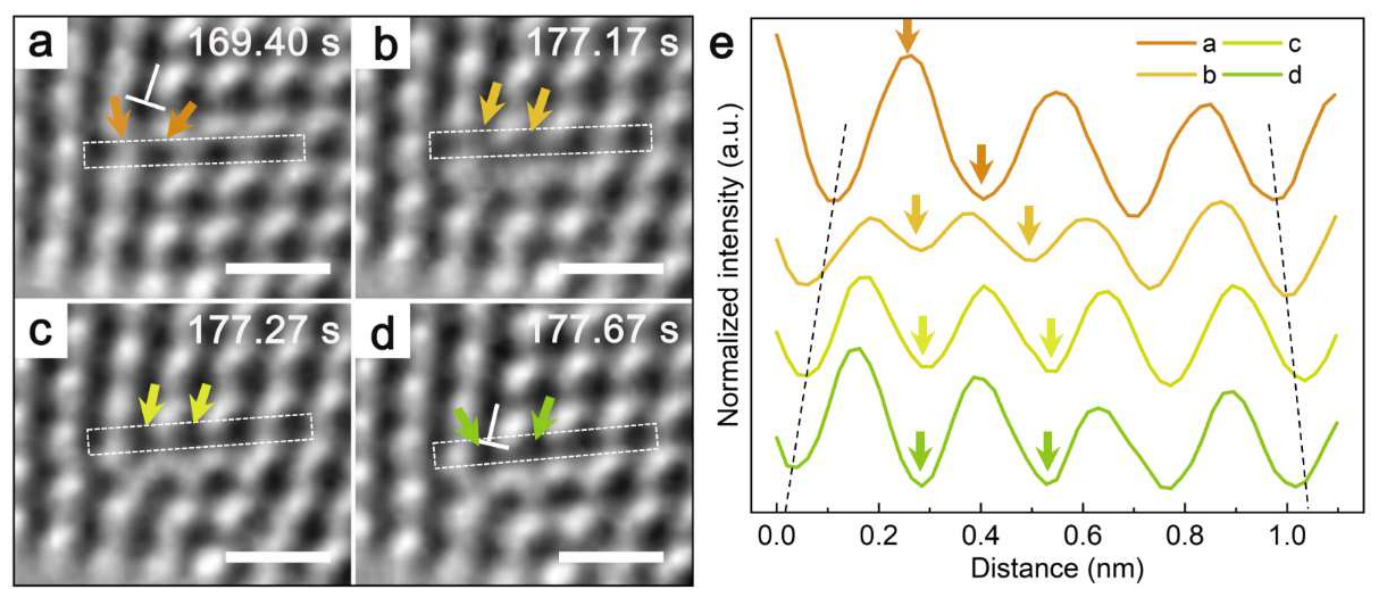

Figure 4. Reconstruction of a dislocation core during negative climb process. (a)-

(d) A series of HRTEM images showing the reconstruction process, with the corresponding contrast intensity profiles in (e) extracted along the dotted rectangle areas in (a)-(d). Scale bar: $0.5 \mathrm{~nm}$. 


\section{Supplementary Files}

This is a list of supplementary files associated with this preprint. Click to download.

- SupplementaryMaterials.docx

- SupplementaryMovie15X.avi

- SupplementaryMovie20.2X.avi

- SupplementaryMovie30.125X.avi

- SupplementaryMovie40.2X.avi

- SupplementaryMovie50.125X.avi 